\title{
Deep-Glassy Ice VI Revealed with a Combination of Neutron Spectroscopy and Diffraction
}

\author{
Alexander Rosu-Finsen, Alfred Amon, Jeff Armstrong, Felix Fernandez-Alonso, \\ and Christoph G. Salzmann*
}

Cite This: J. Phys. Chem. Lett. 2020, 11, 1106-1111

Read Online

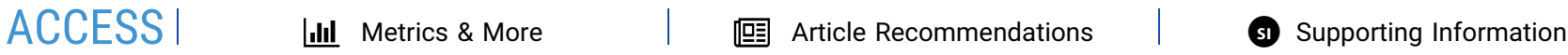

ABSTRACT: The recent discovery of a low-temperature endotherm upon heating hydrochloric-aciddoped ice VI has sparked a vivid controversy. The two competing explanations aiming to explain its origin range from a new distinct crystalline phase of ice to deep-glassy states of the well-known ice VI. Problems with the slow kinetics of deuterated phases have been raised, which we circumvent here entirely by simultaneously measuring the inelastic neutron spectra and neutron diffraction data of $\mathrm{H}_{2} \mathrm{O}$ samples. These measurements support the deep-glassy ice VI scenario and rule out alternative explanations. Additionally, we show that the crystallographic model of $\mathrm{D}_{2} \mathrm{O}$ ice $\mathrm{XV}$, the ordered counterpart of ice VI, also applies to the corresponding $\mathrm{H}_{2} \mathrm{O}$ phase. The discovery of deep-glassy ice VI now provides a fascinating new example of ultrastable glasses that are encountered across a wide range of other materials.

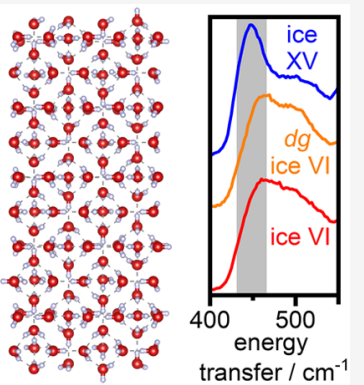

Te is a highly complex material with currently 18 different 1 polymorphs and at least two distinct amorphous forms identified. ${ }^{1,2}$ The highly polar water molecule is capable of forming extended hydrogen-bonded networks with a wide range of different topologies. For a given network, the orientations of the hydrogen-bonded water molecules can adopt, in principle, all configurations from random orientational disorder to fully ordered structures with translation symmetry. ${ }^{1}$ The exothermic phase transitions from so-called hydrogen-disordered phases to their hydrogen-ordered counterparts are expected to take place upon cooling. However, molecular reorientation processes in ice are defect-mediated and hence highly cooperative, which means that orientational glasses can be obtained upon cooling instead of hydrogenordered ices as the reorientation dynamics "freeze-in". This is illustrated, for example, by the differential scanning calorimetry (DSC) scan (1) of hydrogen-disordered ice VI in Figure 1a, which shows a glass transition associated with the unfreezing of molecular reorientations upon heating at $\sim 134 \mathrm{~K}^{3,4}$

A major breakthrough in achieving hydrogen ordering of some of the high-pressure ice phases and, thus, preventing the formation of orientational glasses was the addition of hydrochloric acid $(\mathrm{HCl})$ as a dopant, leading to the discovery of the hydrogen-ordered ices XIII, XIV, and XV. ${ }^{5,6}$ Achieving hydrogen ordering of the various phases of ice is important in order to clarify if their energetic ground states are ferro- or antiferroelectric, ${ }^{7}$ make condensed phases of water available with strictly defined spectroscopic selection rules, ${ }^{8}$ provide benchmark structures for testing the computer models of water, ${ }^{7,9-12}$ and understand geological processes in icy moons and planets. $^{13}$
The formation of ice XV, the hydrogen-ordered counterpart of ice VI, has proven to be one of the most complex cases so far. ${ }^{6,7,10-12,14-17}$ Much of the complexity arises from the fact that ice VI/XV consists of two interpenetrating hydrogenbonded networks. ${ }^{18-20}$ The formation of ice XV therefore goes along with the ordering of the individual networks as well as "internetwork" ordering, which ultimately determines its space group symmetry. ${ }^{6,20,21}$ Intriguingly, the formation of ice $\mathrm{XV}$ is accompanied by an increase in volume that is due to an expansion in the crystallographic $c$ direction. ${ }^{20}$ This means that the formation of ice XV is fastest at low pressures. ${ }^{6,15,21} \mathrm{~A}$ consequence of this is that pressure-quenched samples display an exothermic transient-ordering feature to ice XV upon heating at ambient pressure followed by the disordering phase transition to ice VI. ${ }^{20,21}$ DSC scan (2) in Figure la shows the heating of a highly ordered ice XV sample at ambient pressure that has been obtained by slow-cooling at $0.2 \mathrm{~K} \mathrm{~min}^{-1}$ from $138 \mathrm{~K}^{15,21}$ Figure $1 \mathrm{~b}$ shows a summary of the various possible thermodynamic pathways including the formation of "deepglassy" ice VI if hydrogen ordering can be suppressed, for example, by cooling under pressure. ${ }^{22}$

Cooling $\mathrm{HCl}$-doped ice $\mathrm{VI} / \mathrm{XV}$ at pressures greater than $\sim 1.4 \mathrm{GPa}$ leads to the appearance of a low-temperature endothermic feature before the onset of the transient ordering (cf. scan (3) in Figure 1a). ${ }^{22-24}$ This endotherm has been assigned to the phase transition from a new hydrogen-ordered

Received: January 13, 2020

Accepted: January 23, 2020

Published: January 23, 2020 

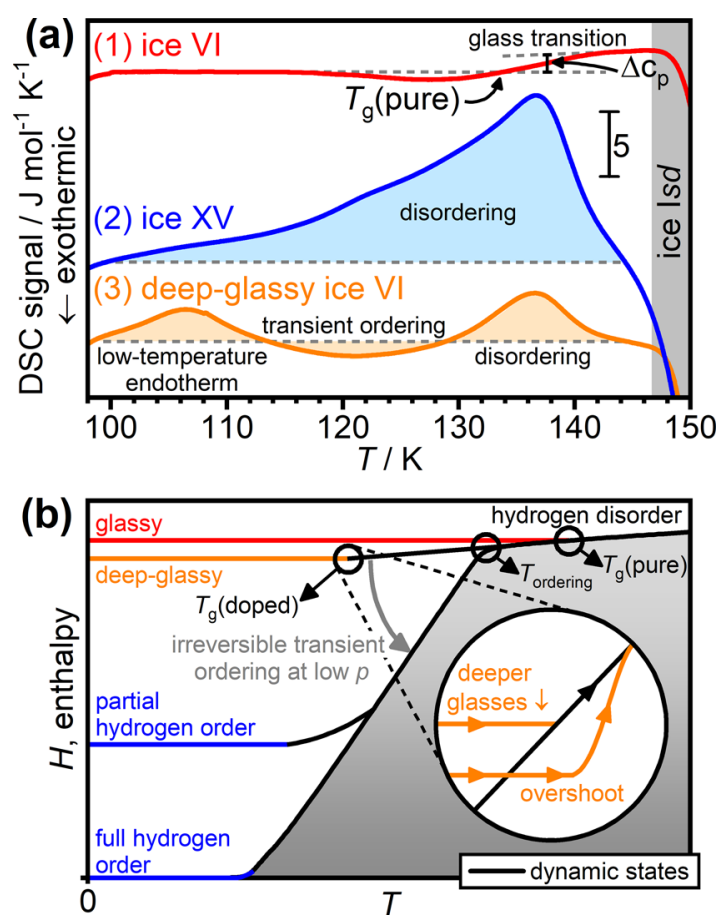

Figure 1. Calorimetry and thermodynamic pathways of pure and $\mathrm{HCl}$-doped ice VI/XV samples. (a) Differential scanning calorimetry scans recorded at $10 \mathrm{~K} \mathrm{~min}^{-1}$ at ambient pressure of (1) pure ice VI quenched at 1.0 GPa, (2) ice XV obtained after slow-cooling $\mathrm{HCl}-$ doped ice VI from $138 \mathrm{~K}$ at $0.2 \mathrm{~K} \mathrm{~min}^{-1}$ at ambient pressure, and (3) deep-glassy $\mathrm{HCl}$-doped ice VI slow-cooled at $1.7 \mathrm{GPa}$ at $0.25 \mathrm{~K}$ $\mathrm{min}^{-1}$. (b) Schematic plot of enthalpy against temperature highlighting the various pathways pure and $\mathrm{HCl}$-doped ice $\mathrm{VI} / \mathrm{XV}$ can follow. The inset shows a magnification of the region around $T_{\mathrm{g}}$ (doped) where deep-glassy ice VI can take different pathways upon heating depending on the state of relaxation.

phase to ice $\mathrm{XV} .^{23}$ According to Gasser et al., their "ice $\beta$-XV" is more hydrogen-ordered, differently hydrogen-ordered, and more stable than ice $\mathrm{XV}{ }^{23}$ Since the new endotherm is irreversible, we pointed out that it is impossible to calculate entropy changes from it and hence to derive direct structural information in terms of hydrogen order. $^{22} \mathrm{~A}$ major misunderstanding in ref 23 is also that the ice $\mathrm{XV}$ to ice VI phase transition does not begin at $129 \mathrm{~K}$ but just above $100 \mathrm{~K}$, as shown in Figure 1a and elsewhere. ${ }^{20,21}$ In contrast to the ice $\beta$-XV scenario, we argued that the low-temperature endotherm arises from the glass transition of deep-glassy ice VI, which becomes increasingly more relaxed or "deeper" as the cooling pressure is increased. ${ }^{22}$ The cooling rate has only a minor effect. Deep-glassy states are well-known to produce endothermic "overshoot" effects ${ }^{25-27}$ upon heating, as shown schematically in the inset of Figure 1b. Accordingly, fast heating rates for a $1.0 \mathrm{GPa}$ cooled sample and prolonged annealing below $T_{\mathrm{g}}$ (doped) at ambient pressure produced the new endotherm as well, and the deep-glassy ice VI scenario is also consistent with the X-ray diffraction and dielectric spectroscopy data in ref $23 .^{22}$ Furthermore, we showed with neutron diffraction that a $\mathrm{D}_{2} \mathrm{O}$ sample that displayed a lowtemperature endotherm has the structure of hydrogendisordered ice VI. ${ }^{22}$ However, it was subsequently argued that the formation of $\mathrm{D}_{2} \mathrm{O}$ ice $\beta$-XV may be hindered for kinetic reasons. $^{28}$
Here we circumvent the potential complications associated with the $\mathrm{D}_{2} \mathrm{O}$ phases entirely, and carry out a combined inelastic neutron spectroscopy and neutron diffraction study of $\mathrm{H}_{2} \mathrm{O}$ samples. In this context, we discuss recently published Raman spectra presented in favor of the ice $\beta$-XV scenario. $^{28}$

For this study, two different $\mathrm{H}_{2} \mathrm{O}$ ice VI/XV samples were prepared: Pure ice VI was obtained by pressure-quenching ice $\mathrm{VI}$ at $1.0 \mathrm{GPa}$ and deep-glassy ice VI by slow-cooling $\mathrm{HCl}$ doped ice VI at $2.5 \mathrm{~K} \mathrm{~min}^{-1}$ at $1.7 \mathrm{GPa}^{22,23}$ The inelastic neutron-scattering (INS) spectra of both samples were then measured at $15 \mathrm{~K}$ on the TOSCA instrument at ISIS $^{29}$ where the deep-glassy ice VI was also transformed to ice XV by heating to $138 \mathrm{~K}$ and subsequent cooling at $0.2 \mathrm{~K} \mathrm{~min}^{-1}$. TOSCA is an indirect neutron spectrometer whose geometry has been specifically optimized for the study of hydrogen motions. ${ }^{29}$ A recent upgrade of this instrument has provided up to a two order of magnitude increase in count rates, thereby enabling parametric neutron-spectroscopic studies alongside simultaneous neutron diffraction.

Figure 2 shows the recorded INS spectra in the low-energytransfer range where modes associated with hindered translations and rotations (librations) of rigid water molecules are observed. It is well-known that this spectral range is particularly sensitive for highlighting the spectroscopic differ-
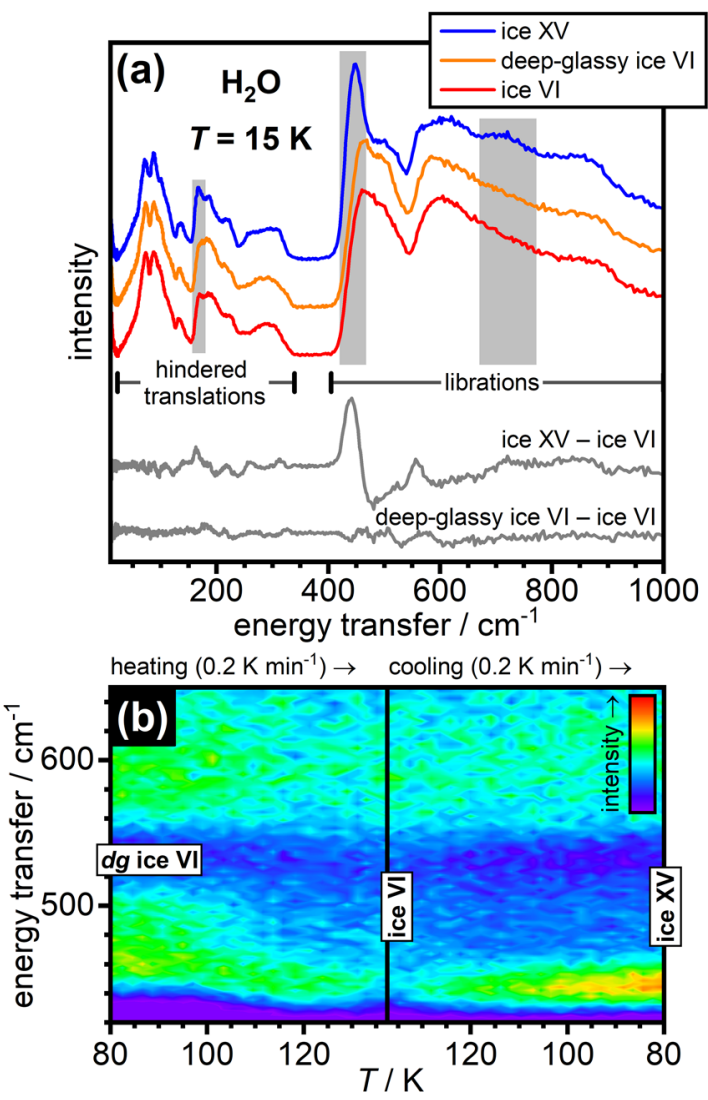

Figure 2. Inelastic neutron spectra of $\mathrm{H}_{2} \mathrm{O}$ ice VI/XV samples. (a) Spectra of ice VI, deep-glassy ice VI, and ice XV collected at $15 \mathrm{~K}$. The gray-shaded areas highlight the spectral range where major differences between the ice VI samples and ice XV were observed. The spectra are shifted vertically for clarity. Difference spectra are shown in the lower part of the panel. The entire available spectroscopic range is shown in Figure S1. (b) Contour plot of the librational region upon heating deep-glassy ice VI from 80 to $138 \mathrm{~K}$, followed by cooling back to $80 \mathrm{~K}$. 
ences between hydrogen-disordered ices and their hydrogenordered counterparts. ${ }^{8,30,31}$ The recorded spectrum of ice VI is consistent with previous reports in the literature ${ }^{32,33}$ and significantly different from the ice $\mathrm{XV}$ spectrum, which is reported here for the first time. Compared to ice VI, ice XV displays additional sharp features at 168 and $448 \mathrm{~cm}^{-1}$, and a broad feature at $\sim 715 \mathrm{~cm}^{-1}$. The gain in intensity at the librational edge for ice XV is consistent with an earlier Raman spectroscopic study ${ }^{8}$ and has also been seen in the INS spectra of the ice VII/VIII disorder-order pair. ${ }^{34}$

The characteristic ice XV spectroscopic features are missing entirely from the spectrum of deep-glassy ice VI, whose spectrum is very similar to that of ice VI. This shows that the new low-temperature endotherm is indeed associated with the glass transition of deep-glassy ice VI, which is structurally very close to "standard" ice VI. An INS spectrum of ice $\beta$-XV, which has been suggested to be more and differently ordered than ice $\mathrm{XV}{ }^{23}$ would have to display a spectrum markedly different from both ice VI and ice XV. ${ }^{8}$ In the Supporting Information, we calculate a "spectroscopic similarity index" (SSI) of 95\% for the similarity between the spectra of deep-glassy and standard ice VI benchmarked against the spectroscopic differences between ice XV and ice VI. As discussed later in detail, a small deviation from $100 \%$ is required by our deep-glassy ice scenario. The SSI of ice $\beta$-XV, however, would be expected to be close to zero or even negative.

Figure $2 \mathrm{~b}$ shows INS spectra collected upon heating deepglassy ice VI from 80 to $138 \mathrm{~K}$ and cooling back at $0.2 \mathrm{~K}$ $\mathrm{min}^{-1}$. Overall, the intensities of the spectroscopic features decrease upon heating due to the effect of temperature on the Debye-Waller factors. Nevertheless, the transient ordering can be seen from the appearance of the $\sim 448 \mathrm{~cm}^{-1}$ ice XV feature above $100 \mathrm{~K}$ and its subsequent disappearance at $138 \mathrm{~K}$. Slowcooling back to $80 \mathrm{~K}$ leads to significant increases in intensity of this feature, illustrating, in line with earlier studies, ${ }^{20,21}$ that slow-cooling produces a much more ordered ice XV compared to the states accessible during the transient ordering upon heating, which is also consistent with the schematic shown in Figure $1 \mathrm{~b}$.

In addition to the INS data, we also analyzed the diffraction data collected synchronously on the TOSCA instrument. Neutron diffraction (ND) of protiated ice generally suffers from intense background signals due to the strong incoherent scattering properties of ${ }^{1} \mathrm{H} .{ }^{33}$ However, the coherent scattering cross section of ${ }^{1} \mathrm{H}$ is sufficiently large compared to that of oxygen so that the diffraction patterns contain information about the structure of the hydrogen sublattice. ${ }^{35}$ A more detailed discussion including comparisons of simulated $\mathrm{H}_{2} \mathrm{O}$ / $\mathrm{D}_{2} \mathrm{O}$ neutron diffraction patterns is presented in the Supporting Information. The ND patterns of ice VI, deepglassy ice VI, and ice XV at $15 \mathrm{~K}$ are shown in Figure 3.

The ND data of ice VI and deep-glassy ice VI are remarkably similar and both data sets could be fitted well using the $P 4_{2} /$ $n m c$ structural model of ice VI. The ND pattern of ice XV, however, is very different and lacks, for example, a Bragg peak at $\sim 1.84 \AA$ characteristic of the two disordered phases. The Rietveld analysis of the ice XV pattern was performed using the $P \overline{1}$ crystallographic model derived earlier for the corresponding $\mathrm{D}_{2} \mathrm{O}$ phase. ${ }^{6,20}$ The ND data at low $d$-spacing can be regarded as a "fingerprint region" due to the many overlapping Bragg peaks. The good agreements in this part of the ND patterns therefore underpin the validities of the used crystallographic models.

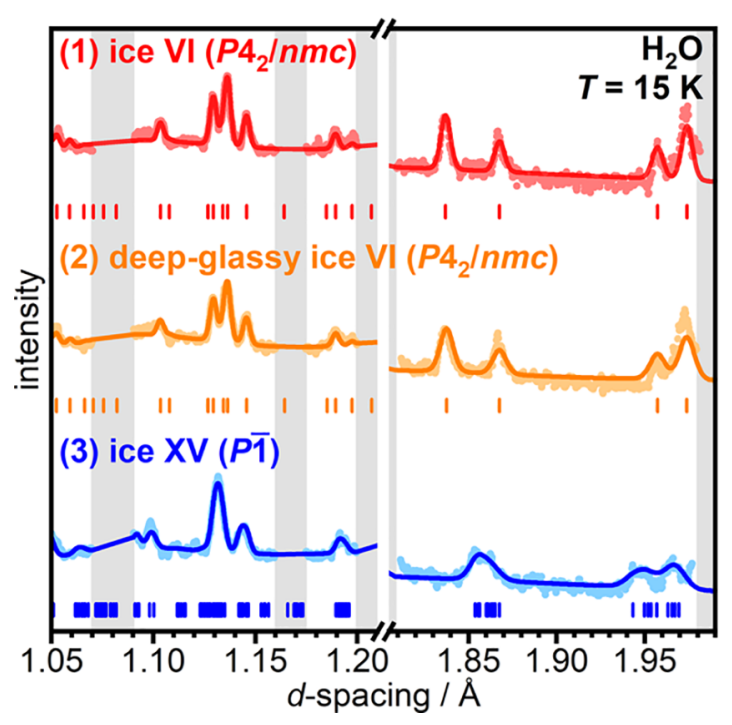

Figure 3. Neutron diffraction patterns of $\mathrm{H}_{2} \mathrm{O}$ (1) ice VI, (2) deepglassy ice VI, and (3) ice XV collected at $15 \mathrm{~K}$. The experimental diffraction data are shown as light data points and the Rietveld fits as darker solid lines. Tick marks indicate the expected positions of Bragg peaks. Gray-shaded areas highlight excluded regions where strong diffraction features from the $\mathrm{Al}$ sample holder were observed. The Rietveld fits over the entire available $d$-spacing range are shown in Figure S4.

As shown in Table 1, the refined lattice constants of deepglassy and "standard" ice VI are the same within the error but very different compared to those of ice $\mathrm{XV}$. The proposed ice $\beta$-XV has been suggested to have a different space group symmetry compared to those of ices VI and $\mathrm{XV}^{23}$ and would therefore have to display a significantly different ND pattern including different lattice constants compared to those of ices VI and XV. More detailed information on the Rietveld analyses, discussion of the lattice constants, and statistical analyses regarding the goodness-of-fit of the diffraction data are given in the Supporting Information.

So far, the crystal structures of the hydrogen-ordered ices were derived from $\mathrm{D}_{2} \mathrm{O}$ phases, and it has been generally assumed that the corresponding $\mathrm{H}_{2} \mathrm{O}$ phases hydrogen-order in similar fashions. The ND data of $\mathrm{H}_{2} \mathrm{O}$ ice XV presented here provide the first evidence for this assumption including similar contractions of the $a$ and $b$ lattice constants and an expansion of $c$ upon ordering.

In summary, it is clear that the "standard" and deep-glassy ice VI are very similar from both the spectroscopic as well as the diffraction point of view, and both are very different from the hydrogen-ordered ice XV. These observations rule out the ice $\beta$-XV scenario $^{23}$ and firmly link the low-temperature endotherm with deep-glassy states of ice VI.

Despite the strong similarities between "standard" and deepglassy ice VI, our scenario requires the two states to be slightly different in enthalpy (cf. Figure 1b). In ref 22 we argued that this enthalpy difference could be realized with very local hydrogen-ordered domains that do not display any orientational correlations between them, so that the average structure is fully hydrogen disordered. Such small domains would then cause strain on the surrounding disordered matrix. Indeed, the Bragg peaks of deep-glassy ice VI are broader than those of "standard" ice $\mathrm{VI}^{22}$ and this effect is also seen for the Bragg peaks at higher $d$-spacing in Figure 3. Taken to the extreme, it 
Table 1. Refined Lattice Constants of $\mathrm{H}_{2} \mathrm{O}$ Ice VI, Deep-Glassy Ice VI, and Ice XV Using Diffraction Data Collected at 15 K on the TOSCA Instrument ${ }^{a}$

\begin{tabular}{lcccccc}
\multicolumn{1}{c}{ sample } & $a / \AA$ & $b / \AA$ & $c / \AA$ & $\alpha / \operatorname{deg}$ & $\beta / \operatorname{deg}$ & $\gamma / \operatorname{deg}$ \\
ice VI & $6.2425(4)$ & $6.2425(4)$ & $5.7667(5)$ & 90 & 90 & 90 \\
deep-glassy ice VI & $6.2421(5)$ & $6.2421(5)$ & $5.7686(8)$ & 90 & 90 & 90 \\
ice XV & $6.2262(7)$ & $6.2105(6)$ & $5.8303(6)$ & $89.90(2)$ & $89.85(2)$ & $89.89(1)$
\end{tabular}

${ }^{a}$ The structural models were based on the $P 4_{2} / n m c$ model of ice $\mathrm{VI}^{18,19}$ and the $P \overline{1}$ model of ice $\mathrm{XV} .{ }^{6,20}$ The numbers in parentheses indicate the errors of the last significant figure.

could be argued that such local distortions could even degrade the space group symmetry of ice VI. At this point it is important to recall that all hydrogen-disordered phases of ice display strictly speaking only $P 1$ space group symmetry due to the lack of translational symmetry. ${ }^{20}$

Compared to results for ice VI, the Raman spectra of what we have assigned to deep-glassy ice VI display small shifts in the $\mathrm{O}-\mathrm{H}$ stretching modes and the high-wavenumber component in the decoupled $\mathrm{O}-\mathrm{D}$ stretching mode region seems to become more Raman active. ${ }^{23,28}$ Changes in the relative intensities of modes in micro-Raman spectroscopy can arise from preferred orientation effects ${ }^{36}$ that clearly play a role, as can be seen from the comparison of nominally identical ice VI/XV materials in the literature. ${ }^{8,23,28,30,37-39}$ However, the shift in the coupled $\mathrm{O}-\mathrm{H}$ stretching mode region may be a sensitive indicator for the strain levels in deep-glassy ice VI. In fact, Raman spectroscopy has been demonstrated to be a very powerful technique for detecting strain in a wide range of different materials. ${ }^{40-42}$ Since Raman intensities arise from changes in the polarizabilities, they can in principle be more sensitive to strain than INS, which directly reflects the vibrational density of states.

The identification of deep-glassy states of ice now adds a fascinating new facet to ice research. In fact, it seems as if deepglassy states and the associated phenomenon of transient ordering have just recently been identified for the "ordinary" ice $\mathrm{I} h$ as well. ${ }^{43}$ Beyond ice, deep-glassy states of natural amber have recently been observed ${ }^{27}$ and the vapor-deposition technique has been shown to be particularly suited for preparing ultrastable glasses with a wide range of scientific challenges and future applications anticipated. ${ }^{44}$ Despite the similar underlying thermodynamics, linking the "deepness" of the glassy states with the details of the local structure will be a highly individual challenge for each material in question.

\section{EXPERIMENTAL METHODS}

Pure $\mathrm{H}_{2} \mathrm{O}$ ice VI samples were obtained by quenching ice VI at $1.0 \mathrm{GPa}$ to $77 \mathrm{~K}$ with liquid nitrogen in a piston-cylinder setup, whereas deep-glassy ice VI was prepared by slow-cooling $0.01 \mathrm{HCl}$-doped $\mathrm{H}_{2} \mathrm{O}$ ice VI at $1.7 \mathrm{GPa}$ at $2.5 \mathrm{~K} \mathrm{~min}^{-1}$ to $77 \mathrm{~K}$. Detailed descriptions of the sample preparations can be found in refs 6, 8, 15, and 20-22. DSC scans of the samples were recorded using a PerkinElmer DSC 8000 as described previously. ${ }^{15,21,22}$ About $3 \mathrm{~g}$ of both samples was ground using a porcelain pestle and mortar under liquid nitrogen and transferred into precooled flat $\mathrm{Al}$ cans with a samplecompartment thicknesses of $2 \mathrm{~mm}$. The sample cans were then mounted onto the cryostat sticks of the helium cryostat of the TOSCA indirect neutron spectrometer at ISIS. ${ }^{29}$ After quick transfers into the cryostat, both samples were measured at $15 \mathrm{~K}$ for at least $2 \mathrm{~h}$. In the case of the deep-glassy ice VI sample, this was followed by heating from 80 to $138 \mathrm{~K}$ at $0.2 \mathrm{~K}$ $\mathrm{min}^{-1}$ and cooling back to $80 \mathrm{~K}$ at $0.2 \mathrm{~K} \mathrm{~min}^{-1}$ to give ice $\mathrm{XV}$ while continuously collecting spectra in 6 min runs. The scattering from the $\mathrm{Al}$ can in the $\pm 45^{\circ}$ spectroscopy banks is negligible in comparison to scattering from ice. TOSCA also contains diffraction detectors in backscattering position at $179^{\circ}$. The ND patterns were analyzed using the GSAS program, ${ }^{45}$ as described in detail in the Supporting Information.

\section{ASSOCIATED CONTENT}

\section{SI Supporting Information}

The Supporting Information is available free of charge at https://pubs.acs.org/doi/10.1021/acs.jpclett.0c00125.

Full-range INS data, calculation of a spectroscopic similarity index, neutron diffraction of $\mathrm{H}_{2} \mathrm{O}$ vs $\mathrm{D}_{2} \mathrm{O}$ ices, full-range neutron diffraction data and Rietveld refinements, changes in the lattice constants, and statistical analyses using the different crystallographic models (PDF)

\section{AUTHOR INFORMATION}

\section{Corresponding Author}

Christoph G. Salzmann - Department of Chemistry, University College London, London WC1H OAJ, United Kingdom; (1) orcid.org/0000-0002-0714-7342; Email: c.salzmann@ ucl.ac.uk

\section{Authors}

Alexander Rosu-Finsen - Department of Chemistry, University College London, London WC1H OAJ, United Kingdom; - orcid.org/0000-0001-5165-7940

Alfred Amon - Department of Chemistry, University College London, London WC1H OAJ, United Kingdom; O orcid.org/ 0000-0002-5301-8867

Jeff Armstrong - ISIS Pulsed Neutron and Muon Source, Rutherford Appleton Laboratory, Didcot OX11 OQX, United Kingdom; ○ orcid.org/0000-0002-8326-3097

Felix Fernandez-Alonso - Department of Physics and Astronomy, University College London, London WC1E 6BT, United Kingdom; Materials Physics Center, CSIC-UPV/EHU, 20018 Donostia, Spain; IKERBASQUE, Basque Foundation for Science, 48013 Bilbao, Spain; 이이.org/0000-00030313-017X

Complete contact information is available at:

https://pubs.acs.org/10.1021/acs.jpclett.0c00125

\section{Notes}

The authors declare no competing financial interest.

\section{ACKNOWLEDGMENTS}

We thank the Royal Society for a University Research Fellowship (CGS, UF150665), the Austrian Science Fund for a Schrödinger fellowship (AA, J4325), and ISIS for granting 
access to the TOSCA instrument. Furthermore, this project has received funding from the European Research Council (ERC) under the European Union's Horizon 2020 research and innovation program (grant agreement no. 725271).

\section{REFERENCES}

(1) Salzmann, C. G. Advances in the Experimental Exploration of Water's Phase Diagram. J. Chem. Phys. 2019, 150, 060901.

(2) Millot, M.; Coppari, F.; Rygg, J. R.; Correa Barrios, A.; Hamel, S.; Swift, D. C.; Eggert, J. H. Nanosecond X-ray Diffraction of Shockcompressed Superionic Water Ice. Nature 2019, 569, 251-255.

(3) Handa, Y. P.; Klug, D. D.; Whalley, E. Energies of the Phases of Ice at Low Temperature and Pressure Relative to Ice I $h$. Can. J. Chem. 1988, 66, 919-924.

(4) Shephard, J. J.; Salzmann, C. G. Molecular Reorientation Dynamics Govern the Glass Transitions of the Amorphous Ices. J. Phys. Chem. Lett. 2016, 7, 2281-2285.

(5) Salzmann, C. G.; Radaelli, P. G.; Hallbrucker, A.; Mayer, E.; Finney, J. L. The Preparation and Structures of Hydrogen Ordered Phases of Ice. Science 2006, 311, 1758-1761.

(6) Salzmann, C. G.; Radaelli, P. G.; Mayer, E.; Finney, J. L. Ice XV: A New Thermodynamically Stable Phase of Ice. Phys. Rev. Lett. 2009, 103, 105701.

(7) Del Ben, M.; VandeVondele, J.; Slater, B. Periodic MP2, RPA, and Boundary Condition Assessment of Hydrogen Ordering in Ice XV. J. Phys. Chem. Lett. 2014, 5, 4122-4128.

(8) Whale, T. F.; Clark, S. J.; Finney, J. L.; Salzmann, C. G. DFTassisted Interpretation of the Raman Spectra of Hydrogen-ordered Ice XV. J. Raman Spectrosc. 2013, 44, 290-298.

(9) Singer, S. J.; Knight, C. Hydrogen-Bond Topology and Proton Ordering in Ice and Water Clusters. Advances in Chemical Physics; John Wiley \& Sons, Inc., 2011; pp 1-74.

(10) Kuo, J.-L.; Kuhs, W. F. A First Principles Study on the Structure of Ice-VI: Static Distortion, Molecular Geometry, and Proton Ordering. J. Phys. Chem. B 2006, 110, 3697-3703.

(11) Knight, C.; Singer, S. J. Prediction of a Phase Transition to a Hydrogen Bond Ordered Form of Ice VI. J. Phys. Chem. B 2005, 109, 21040-21046.

(12) Nanda, K. D.; Beran, G. J. O. What Governs the Proton Ordering in Ice XV? J. Phys. Chem. Lett. 2013, 4, 3165-3169.

(13) Mitra, S. High-pressure Geochemistry and Mineral Physics; Elsevier: Amsterdam, The Netherlands, 2004.

(14) Komatsu, K.; Noritake, F.; Machida, S.; Sano-Furukawa, A.; Hattori, T.; Yamane, R.; Kagi, H. Partially Ordered State of Ice XV. Sci. Rep. 2016, 6, 28920.

(15) Rosu-Finsen, A.; Salzmann, C. G. Benchmarking Acid and Base Dopants with Respect to Enabling the Ice V to XIII and Ice VI to XV Hydrogen-ordering Phase Transitions. J. Chem. Phys. 2018, 148, 244507.

(16) Johari, G. P.; Whalley, E. Evidence for a Very Slow Transformation in Ice VI at Low Temperatures. J. Chem. Phys. 1979, 70, 2094-2097.

(17) Mishima, O.; Mori, N.; Endo, S. Thermal expansion anomaly of ice VI related to the order-disorder transtion. J. Chem. Phys. 1979, 70, 2037-2038.

(18) Kamb, B. Structure of Ice VI. Science 1965, 150, 205-209.

(19) Kuhs, W. F.; Finney, J. L.; Vettier, C.; Bliss, D. V. Structure and Hydrogen Ordering in Ices VI, VII, and VIII by Neutron Powder Diffraction. J. Chem. Phys. 1984, 81, 3612-3623.

(20) Salzmann, C. G.; Slater, B.; Radaelli, P. G.; Finney, J. L.; Shephard, J. J.; Rosillo-Lopez, M.; Hindley, J. Detailed Crystallographic Analysis of the Ice VI to Ice XV Hydrogen Ordering Phase Transition. J. Chem. Phys. 2016, 145, 204501.

(21) Shephard, J. J.; Salzmann, C. G. The Complex Kinetics of the Ice VI to Ice XV Hydrogen Ordering Phase Transition. Chem. Phys. Lett. 2015, 637, 63-66.

(22) Rosu-Finsen, A.; Salzmann, C. G. Origin of the Lowtemperature Endotherm of Acid-doped Ice VI: New Hydrogen- ordered Phase of Ice or Deep Glassy States? Chem. Sci. 2019, 10, 515-523.

(23) Gasser, T. M.; Thoeny, A. V.; Plaga, L. J.; Köster, K. W.; Etter, M.; Böhmer, R.; Loerting, T. Experiments Indicating a Second Hydrogen Ordered Phase of Ice VI. Chem. Sci. 2018, 9, 4224-4234.

(24) Sharp, S. Study challenges hydrogen-ordered ice hypothesis. https://www.chemistryworld.com/news/study-challenges-hydrogenordered-ice-hypothesis/3010088.article.

(25) Moynihan, C. T.; Macedo, P. B.; Montrose, C. J.; Montrose, C. J.; Gupta, P. K.; DeBolt, M. A.; Dill, J. F.; Dom, B. E.; Drake, P. W.; Easteal, A. J.; et al. Structural Relaxation in Vitreous Materials. Ann. N. Y. Acad. Sci. 1976, 279, 15-35.

(26) Höhne, G.; Hemminger, W.; Flammersheim, H.-J. Differential Scanning Calorimetry - An Introduction for Practitioners; Springer: Berlin, 1996.

(27) Zhao, J.; Simon, S. L.; McKenna, G. B. Using 20-million-Yearold Amber to Test the Super-Arrhenius Behaviour of Glass-forming Systems. Nat. Commun. 2013, 4, 1783.

(28) Thoeny, A. V.; Gasser, T. M.; Loerting, T. Distinguishing Ice $\beta$ XV from Deep Glassy Ice VI: Raman Spectroscopy. Phys. Chem. Chem. Phys. 2019, 21, 15452-15462.

(29) Pinna, R. S.; Rudić, S.; Parker, S. F.; Armstrong, J.; Zanetti, M.; Koro, G.; Waller, S. P.; Zacek, D.; Smith, C. A.; Capstick, M. J.; et al. The Neutron Guide Upgrade of the TOSCA Spectrometer. Nucl. Instrum. Methods Phys. Res., Sect. A 2018, 896, 68-74.

(30) Minceva-Sukarova, B.; Sherman, W. F.; Wilkinson, G. R. The Raman Spectra of Ice ( $\mathrm{I}_{\mathrm{h}}$, II, III, V, VI and IX) as Functions of Pressure and Temperature. J. Phys. C: Solid State Phys. 1984, 17, $5833-5850$.

(31) Minceva-Sukarova, B.; Slark, G. E.; Sherman, W. F.; Wilkinson, G. R. A Re-correlation of the Vibrational Spectra and Crystallographic Data for the Various Ice Polymorphs. J. Phys. (Paris) 1987, 48, 37-43 Colloque $\mathrm{C} 1$.

(32) Li, J.-C.; Londono, J. D.; Ross, D. K. An inelastic incoherent neutron scattering study of ice II, IX, V, and VI - in the range from 2 to $140 \mathrm{meV}$. J. Chem. Phys. 1991, 94, 6770-6775.

(33) $\mathrm{Li}, \mathrm{J}$. Inelastic neutron scattering studies of hydrogen bonding in ices. J. Chem. Phys. 1996, 105, 6733-6755.

(34) Klotz, S.; Strässle, T.; Salzmann, C. G.; Philippe, J.; Parker, S. F. Incoherent Inelastic Neutron Scattering Measurements on Ice VII: Are There Two Kinds of Hydrogen Bonds in Ice? Europhys. Lett. 2005, 72, 576-582.

(35) Sears, V. F. Neutron Scattering Lengths and Cross Sections. Neutron News 1992, 3, 26-37.

(36) Salzmann, C. G.; Kohl, I.; Loerting, T.; Mayer, E.; Hallbrucker, A. Raman Spectroscopic Study on Hydrogen Bonding in Recovered Ice IV. J. Phys. Chem. B 2003, 107, 2802-2807.

(37) Gasser, T. M.; Thoeny, A.; Plaga, L.; Köster, K. W.; Etter, M.; Böhmer, R.; Loerting, T. Experimental Evidence for a Second Hydrogen ordered Phase of Ice VI. arXiv 1708.06601 [cond-mat.mtrlsci] 2017.

(38) Minceva-Sukarova, B.; Slark, G. E.; Sherman, W. F. The Raman spectra of ice $\mathrm{V}$ and ice VI and evidence of partial proton ordering at low temperature. J. Mol. Struct. 1986, 143, 87-90.

(39) Minceva-Sukarova, B.; Slark, G.; Sherman, W. F. The Raman Spectra of the KOH-doped Ice Polymorphs: V and VI. J. Mol. Struct. 1988, 175, 289-293.

(40) Lee, T.; Mas'ud, F. A.; Kim, M. J.; Rho, H. Spatially Resolved Raman Spectroscopy of Defects, Strains, and Strain Fluctuations in Domain Structures of Monolayer Graphene. Sci. Rep. 2017, 7, 16681.

(41) Cheng, T.; Tan, C.; Zhang, S.; Tu, T.; Peng, H.; Liu, Z. Raman Spectra and Strain Effects in Bismuth Oxychalcogenides. J. Phys. Chem. C 2018, 122, 19970-19980.

(42) Tuschel, D. Stress, Strain, and Raman spectroscopy. Spectroscopy 2019, 34, 10-21.

(43) Fortes, A. D. Structural Manifestation of Partial Proton Ordering and Defect Mobility in Ice Ih. Phys. Chem. Chem. Phys. 2019, 21, 8264-8274. 
(44) Ediger, M. D. Perspective: Highly Stable Vapor-deposited Glasses. J. Chem. Phys. 2017, 147, 210901.

(45) Larsen, A. C.; Von Dreele, R. B. GSAS - General Structure Analysis System; University of California, 1985. 